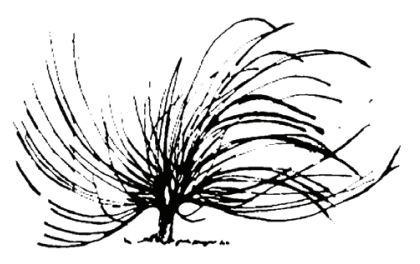

\title{
Efectividad del programa ecológico "Saltando por su salud" en la promoción de la actividad física y la auto-eficacia en niños y niñas escolares de tercer grado
}

\author{
Carlos Álvarez Bogantes ${ }^{1}$ \\ Universidad Nacional \\ Heredia, Costa Rica \\ ceab.03@gmail.com
}

\begin{abstract}
Resumen
El propósito de este artículo fue el de evaluar el efecto del Programa Saltando por su salud sobre las variables de actividad física y auto-eficacia en niños y niñas de tercer grado usando un modelo ecológico escolar. Diseño del estudio: Un diseño cuasi-experimental utilizado a través de 4 meses. Sujetos: tomaron parte en el estudio 34 niños y 32 niñas con edades promedio de 9.2 años, con un $30 \%$ de muestra de participantes con sobrepeso u obesidad. Intervención: estructuración de la clase de educación física para fomentar la actividad física moderada, la enseñanza de actividades físicas para la vida y destrezas conductuales, junto con el desarrollo de las condiciones ambientales y apoyo social en el recreo para la realización de actividad física. Finalmente, el desarrollo de políticas institucionales en el marco de convertir la escuela en una escuela en movimiento. Resultados: Los niños y niñas en el grupo de intervención tuvieron un incremento significativo en la
\end{abstract}

\section{(c) (i) (8) $\Theta$}

Recibido: 29 de febrero de 2016-Aprobado: 25 de abril de 2016

1 Promotor de la Salud Fisica, Doctor en Educación, UNED 
actividad física $(\mathrm{p}<.001)$ medido por podómetros. Además, mostraron un mejoramiento en el factor de superación de barreras para realizar actividad física $(\mathrm{p}<.001)$. Conclusiones: El programa Saltando por su salud mejoró el tiempo dedicado a la actividad física y a superar barreras para realizar actividad física Este estudio demostró que usando los recursos existentes institucionales escolares y mediante un acercamiento ecológico, se pueden propiciar entornos saludables en movimiento en el contexto educativo.

Palabras clave: Educación física, actividad física moderada, niños y niñas, recreo escolar, apoyo social, destrezas conductuales, manejo de barreras ambientales.

\begin{abstract}
The purpose of this paper was to evaluate the effectiveness of the "Saltando por su Salud" program on the variables of physical activity and self-efficacy in third grade School children, using an ecological model. Study Design: A quasi-experimental design was used for 4 months. Subject: 34 boys and 32 girls took part with an average age of 9.2 years. $30 \%$ of the participants were overweight or obese. Intervention: the intervention included: structuring of PE to encourage moderate physical activity, physical education activities for life and behavioral skills, along with the development of environmental conditions and social support to promote physical activity during recess. Results: Children in the intervention group had a significant increase in physical activity ( $p$ $<.001)$ measured by pedometers. In addition, they showed an improvement in the seft-efficacy to overcome barriers for physical activity $(\mathrm{p}<.001)$. Conclusions: The "Saltando por su Salud" program improved time spent on physical activity and strategies to overcome barriers for physical activity This study demonstrated that using existing school resources and an ecological approach, It is possible to build healthier school environments.
\end{abstract}

Keywords: Physical Education, moderate physical activity, children, school recreation, social support, behavioral skills, management of environmental barriers 


\section{Introducción}

Los beneficios físicos, mentales y sociales de la actividad física para la niñez son ampliamente reconocidos (Borehan \& Riddoch, 2001; Wing et al., 2001). Sin embargo, a pesar de las recomendaciones de salud pública, para que los niños y niñas realicen por lo menos 60 minutos cada día en moderada a vigorosa actividad física de intensidad (Strong et al., 2005), la población infantil cada vez muestra mayores niveles de sedentarismo, lo que la expone a un mayor riesgo de desarrollar sobrepeso u obesidad (Biddel, Gorely \& Stensel, 2004; Wareham et al., 2005) y a enfermedades como la diabetes y las enfermedades cardiovasculares (Strong et al, 2005; WHO, 2002). Esto es especialmente preocupante cuando hablamos de los niños y niñas que presentan sobrepeso y obesidad, ya que la disminución de los niveles de actividad física desde la infancia a través de la adolescencia es más evidente enesta población, lo que requiere estrategias de intervención efectivas y eficaces durante la infancia para contribuir a una niñez más activa y saludable (Janz, Burns and Levy, 2005).

Muchas intervenciones con el objetivo de aumentar la actividad física en el entorno escolar se han desarrollado e implementado en una variedad de países (Salmon et al., 2007; Stone et al., 1998). Estos estudios han mostrado una gran eficiencia en la disminución de factores de riesgo de enfermedades degenerativas, y en la disminución de los niveles de sedentarismo en el entorno de la clase de educación física (Kahn et al, 2002; Stone et al, 1998); sin embargo, es difícil encontrar intervenciones que se hayan ejecutado con estudiantes de primaria integrando a los niños y niñas con sobrepeso y obesidad en los entornos de la escuela, con el fin de incrementar la actividad física de sus estudiantes.

En el marco de la prevención en salud en niños y niñas con sobrepeso y obesidad, se ha aceptado que el acercamiento más apropiado a la hora de desarrollar intervenciones para disminuir los niveles de sedentarismo en niños y niñas es el modelo que se ejecuta en el contexto educativo integrando a toda la población, ya que los niños y niñas ya presentan una prevalencia y un riesgo alto para sobrepeso y obesidad. Adicionalmente, los programas e intervenciones de promoción de salud escolar y de intervención en las poblaciones escolares, que son específicos a poblaciones con sobrepeso u obesidad, son difíciles de sostener en el tiempo, presentando un impacto muy reducido y niveles de efectividad cuestionables (Goran, Reynolds \& Lindquist, 1999; Kumanyika, 2001). 
Evidentemente, considerar las escuelas como los espacios fundamentales, para que niños y niñas puedan ser activos y construyan las destrezas necesarias para enfrentar una cultura sedentaria, tiene todo el potencial para promover estilos de vida en movimiento. Hay acuerdo en que el enfocarse en estos entornos para facilitar la actividad física es una de las estrategias que ofrece el mayor rigor para impactar a los niños y niñas hacia una vida futura en movimiento (Salmon, 2007), cuando se toma en cuenta los factores que influencian las conductas que conducen al cambio hacia estilos de vida en movimiento.

En respuesta a la importancia de la escuela como centro de promoción y prevención del sobrepeso y la obesidad, surge la educación física, como ente coordinador de esos esfuerzos sostenibles de una niñez en movimiento en los centros educativos (Cox, Schofield \& Kold, 2010; Salmon et al., 2007; Timperio et al., 2004)

Se propone la educación física como la materia escolar que presenta el potencial para contemplar múltiples esferas de influencia, con un enfoque socio-ecológico, que centra sus esfuerzos en las relaciones entre los individuos y el entorno social, físico y político, reconociéndose que la actividad física en la niñez está determinada por una interacción compleja de factores en diferentes niveles, incluyendo el nivel intrapersonal y la auto-eficacia, el ambiente social y ambiental (Ward, Saunders $\&$ Pate, 2007). Desde el contexto de las clases de educación física a nivel escolar, se puedan desarrollar propuestas que incluyan una estructura de clase que garantice actividades de moderada intensidad durante al menos un $40 \%$ del tiempo, el aprendizaje de destrezas físicas y conductuales que provean las herramientas para que la persona sea activa, y el desarrollo de entornos y políticas educativas que brinden las posibilidades para activar a los niños y niñas de por vida (Ward et al., 2007).

Considerando lo anterior, se plantea el objetivo de determinar el efecto de un programa de consejería en actividad física grupal sobre los patrones de movimiento y la auto-eficacia de niños y niñas de una escuela pública con el uso de estrategias que fomenten la actividad física de moderada intensidad, la enseñanza de actividades físicas para la vida y destrezas conductuales en el contexto escolar.

Partiendo de lo anterior, se plantea el objetivo de determinar el efecto del programa Ecológico "Saltando por su salud" sobre la actividad física y la auto-eficacia de niños y niñas de una escuela pública, usando estrategias que fomenten la actividad física moderada, la 
enseñanza de actividades físicas para la vida y destrezas conductuales durante la clase de educación física, junto con el desarrollo de las condiciones ambientales y apoyo social en el recreo para la realización de actividad física. Finalmente, se promovieron políticas institucionales en el marco de convertir la escuela en una escuela en movimiento.

\section{Metodología}

\section{Participantes}

Los participantes en este estudio fueron 59 estudiantes de tercer grado de una escuela urbana pública del cantón de Belén de Heredia, de los cuales 35 pertenecieron al grupo experimental (19 niños y 16 niñas) y 36 al grupo control (15 niños y 16 niñas). Para responder al objetivo de esta investigación y siguiendo las recomendaciones de la CCSS, rel estudiantado seleccionado perteneció a dos de las secciones de tercer grado que presentaron los niveles más altos de sobrepeso y obesidad, seleccionados de la evaluación del IMC total de estudiantes de la institución.

\section{Instrumentos}

Para calcular la variable de pasos realizados, se utilizaron podómetros Omron HJ-720 ITC. Según Tudor-Locke, MaClain, Hart, Sisson y Washington (2009), este monitor posee correlaciones intraclase durante 8 días $(0,87)$, los días entre semana $(0.75)$, los días escolares $(0,74)$ y los días de fin de semana $(0,59)$. Este es un instrumento exacto para medir la cantidad de pasos (Giannakidou et al., 2012) y ofrece un porcentaje de error absoluto de $<3.0 \%$ (Holbrook, Barreira, \& Kang, 2009). El podómetro Omron HJ-720 ITC ha demostrado tener una aceptable validez y confiabilidad para medir la cantidad de pasos en la edad infantil (Lee et al., 2012; Peters, Heelan, \& Abbey, 2013) y el rango de error de 50 podómetros no excedió el $\pm 5 \%$. Esto se realizó para conocer con más exactitud, si los estudiantes se mantienen en constante movimiento durante su horario lectivo.

Además para medir la variable autoeficacia, se realizó el cuestionario de autoeficacia hacia la actividad física en niños y niñas (Aedo \& Ávila, 2009), validado con una consistencia alfa de Cronbach de 0,733 y 
la confiabilidad test-retest de 0,867 ; lo cual nos da una escala con validez y confiabilidad adecuadas que permiten utilizarla como un buen indicador de la autoeficacia hacia la actividad física en niños y niñas de edad escolar.

\section{Procedimientos}

\section{Aspectos administrativos}

En la primera fase de este proyecto y contando con el aval del MEP, se conformó un equipo técnico con el Ministerio de Salud, la CCSS, la Escuela seleccionada y la UNA, para realizar la evaluación del IMC de todos sus estudiantes y de apoyar la intervención "Saltando por tu salud". Adicionalmente a este grupo, se conformó el grupo administrativo, conformado por el orientador de la escuela seleccionada y el coordinador del programa "Saltando por su salud" de la UNA.

Junto con la CCSS, se realizó la evaluación del IMC de la totalidad de estudiantes de la institución, no solo para cumplir los requerimientos del programa de Salud escolar de la CCSS, sino también para poder escoger el nivel y secciones que participarían en la intervención "Saltando por su salud". Siguiendo el criterio de seleccionar el nivel y las secciones que presentaran mayor incidencia de sobrepeso y obesidad, se seleccionaron dos terceros grados para que fueran el grupo control y el experimental, respectivamente.

Una vez obtenido el consentimiento informado por parte de las familias de participantes en la intervención, el comité administrativo procedió a realizar una visita al centro educativo para explicar y coordinar con el director, las maestras de grado y los educadores físicos los aspectos administrativos de la investigación y el cronograma de actividades de las intervención de cuatro meses.

Una vez seleccionado el grupo a intervenir y el grupo control, los datos del pretest y postest fueron obtenidos durante la clase de educación del grupo seleccionado, con ayuda del educador físico del grupo, en la variable la autoeficacia. Con respecto a la variable dependiente de pasos, estudiantes y maestra recibieron una inducción del uso del podómetro. Los podómetros los usaron durante dos días en el espacio de la jornada académica de la escuela que es de 7 de la mañana a 2 de la tarde. Esta medición de pasos se realizó durante dos días, en la jornada escolar. 


\section{Intervención "Saltando por nuestra salud"}

El programa de intervención "Saltando por nuestra salud" fue una propuesta en una escuela pública del cantón de Belén con un duración de 4 meses, y con una periodicidad de una vez por semana durante la clase de educación física y en los recreos escolares, administrada desde el ámbito de las clases de educación física. Como elemento conceptual para el desarrollo de esta intervención, se utilizó la teoría ecológica de cambio de conducta (Ward et al, 2007), especialmente en los niveles personales, interpersonales y organizacionales. Este programa utilizó a nivel personal los constructos de autoeficacia, destrezas físicas y conductuales; en el nivel interpersonal se utilizó al profesor de educación física y a las maestras como motivadores para incrementar la actividad física durante las clases de educación física y en los recreos escolares. Finalmente, a nivel organizacional, el director del centro creó la comisión de actividad física, encargada de designar espacios y eliminar barreras físicas dentro de la institución para que los niños y niñas intervenidos pudieran jugar libremente, con el material para realizar actividad física que la maestra administraba.

\section{Diseño y análisis}

Se realizó la estadística descriptiva calculando los promedios y las desviaciones estándar de las variables dependientes. Se utilizó un diseño cuasi-experimental, siguiendo un diseño de pretest-postest con grupos control y experimental. Para determinar si hubo diferencias significativas se utilizó una prueba ANOVA factorial 2 X 2 . Al encontrarse diferencias significativas en ambas variables, se aplicó un test post hoc, para determinar donde se dieron las diferencias. Con respecto a la variable de actividad física se realizó una regresión múltiple con el método de pasos sucesivos, teniendo como variable dependiente la diferencia (post-pre), entre las mediciones de pasos, para saber si el índice de masa corporal pretest y el sexo de los sujetos incidieron en los resultados.

\section{Resultados}

Los respectivos IMC de los niños y niñas con peso normal fueron $16.02( \pm 0.12)$ y $15.83( \pm 0.13) \mathrm{Km} / \mathrm{m}^{2}$. El grupo de niños y niñas 
con sobrepeso y obesidad representó un $34 \%$ del total de participantes, donde el IMC de los niños y niñas con obesidad y sobrepeso fue de $21.33(0.27)$ y de $21.04(0.31) \mathrm{Km} / \mathrm{m} 2$, respectivamente.

Los promedios y las desviaciones estándar de la variable dependiente de actividad física son presentadas en la tabla 1. En la tabla 2 se muestra el resultado del análisis de varianza de dos vías de la variable dependiente de actividad física. Los resultados indican que el comportamiento de los dos grupos entre ambas mediciones fue diferente dado que se encontró interacción significativa entre grupo y mediciones $(F$ : 85.16; sig.<.001). De acuerdo al análisis post hoc de efectos simples el grupo control no cambio significativamente entre mediciones, mientras que el grupo experimental si cambió y lo hizo aumentando significativamente su promedio de pasos. De acuerdo con los resultados del análisis de varianza, se evidencia que la pertenencia al grupo experimental puede favorecer aumentos en la cantidad de pasos; para saber si el índice de masa corporal pre test y el sexo de los sujetos inciden en estos resultados, se procedió a realizar una regresión múltiple con el método de pasos sucesivos, teniendo como variable dependiente la diferencia (post-pre), entre las mediciones de pasos.

Tabla 1. Resumen de análisis descriptivos de la variable actividad física medida en niños y niñas de tercer grado de la Escuela Fidel Chaves

\begin{tabular}{|l|l|c|c|c|}
\hline \multirow{3}{*}{ Pretest } & \multicolumn{1}{|c|}{ Grupo } & Media & Desviación típica & n \\
\cline { 2 - 5 } & Control & $3,635.51$ & 612.574 & 30 \\
\cline { 2 - 5 } & Experi. & $3,235.34$ & 846.970 & 31 \\
\cline { 2 - 5 } & Total & $3,435.43$ & 759.9040 & 61 \\
\hline Postest & Control & $3,604.72$ & 629,088 & 30 \\
\hline & Experi & $4,039.72$ & 828,878 & 61 \\
\hline
\end{tabular}


Tabla 2. Resumen de análisis de varianza de dos vías (medición $x$ grupo) de la variable actividad física medidas en niños y niñas de tercer grado de la Escuela Fidel Chaves

\begin{tabular}{|l|c|c|}
\hline \multicolumn{1}{|c|}{ Fuente de varianza verdadera } & F & Sig. \\
\hline Medición & 73,067 & $<0,001$ \\
\hline Medición * grupo & 85,165 & $<0,001$ \\
\hline Grupo & 0,009 & 0,027 \\
\hline
\end{tabular}

La regresión fue significativa $\left(\mathrm{R}^{2}: 0.603 ; F: 85.16\right.$; sig.<.001), predictora significativa, mientras que las variables sexo (1: mujer; o: hombre) y IMC (1: sobrepesouobesidad; 0: normal) no fueron predictoras significativas de la diferencia de pasos obtenida en el postest. Es decir, el grupo al cual pertenece un sujeto explica un $60.3 \%$ de la diferencia de pasos entre mediciones y que el pertenecer al grupo experimental implica un aumento de 835.17 pasos.

Los promedios y las desviaciones estandar de la variable dependiente del factor de superación de barreras se presenta en la tabla 3. En la tabla 4 se muestra los resultados del análisis de varianza.

Tabla 3. Resumen de análisis descriptivos del factor de superación de barreras de la variable de autoeficacia medida en niños y niñas de tercer grado de la Escuela Fidel Chaves

\begin{tabular}{|l|l|c|c|c|}
\hline & Grupo & Media & Desviación típica & $\mathbf{n}$ \\
\hline Pretest & Control & 1,3931 &, 30464 & 30 \\
\cline { 2 - 5 } & Experi. & 1,4621 &, 27828 & 31 \\
\cline { 2 - 5 } & Total & 1,4276 &, 29127 & 61 \\
\hline Postest & Control & 1,4138 &, 30205 & 30 \\
& Experi & 1,2759 &, 18833 & 31 \\
& Total & 1,3448 &, 25899 & 61 \\
\hline
\end{tabular}


Tabla 4. Resumen de análisis de varianza de dos vías (medición $\mathrm{x}$ grupo) del factor de superación de barreras de la variable de autoeficacia medida en niños y niñas de tercer grado de la Escuela Fidel Chaves

\begin{tabular}{|l|c|c|}
\hline \multicolumn{1}{|c|}{ Fuente de varianza verdadera } & F & Sig. \\
\hline Medición & 11,947 & $<0,001$ \\
\hline Medición * grupo & 18,667 & $<0,001$ \\
\hline Grupo &, 262 & 0,611 \\
\hline
\end{tabular}

Con respecto al constructo de autoeficacia, solo se encontraron efectos significativos del programa Saltando por su salud, en el factor de barreras, debido a que solo en esta variable se encontró interacción entre grupos y mediciones. El comportamiento de los dos grupos entre ambas mediciones fue diferente, dado que se encontró interacción significativa entre grupo y mediciones $(F: 18.667 ;$ sig. $<.001)$.

De acuerdo con el análisis post hoc de efectos simples, el grupo control no cambio significativamente entre mediciones, mientras que el grupo experimental sí cambio y lo hizo disminuyendo significativamente su promedio en el factor de superación de barreras. Sin embargo, también el análisis post hoc mostró que existían diferencias significativas entre ambos grupos tanto en el pretest como en el postest. De acuerdo con los resultados del análisis de varianza se evidencia que la pertenencia al grupo experimental puede favorecer mejoramientos en el factor de superación de barreras de la variable de auto-eficacia.

\section{Discusión}

Desde una perspectiva de la promoción de la actividad física para la salud, este estudio ha proporcionado información útil, que muestra que las intervenciones escolares pueden ser efectivas, cuando se entienden los mecanismos a través de los cuales las intervenciones logran éxito en cambiar la conducta sedentaria, respondiendo al contexto educativo, a la cultura institucional y a las posibilidades reales de aplicación siguiendo un modelo ecológico.

Hay dos resultados en esta investigación que deben enfatizarse. Primero, la puntuación media de número de pasos realizados durante la jornada escolar del grupo experimental aumentó significativamente 
en relación con la línea de base y al grupo control. Segundo, que el componente de superación de las barreras en el constructo o mediador de auto-eficacia se mejoró significativamente con respecto a la línea base y al grupo control; sin embargo, los componentes de dimensión de alternativas y expectativas del constructo de autoeficacia no fueron modificados, lo que sugiere que la intervención fue efectiva en la mejora del autocontrol de sus participantes en visualizar y disminuir los elementos ambientales que frenan la participación en actividad física a nivel escolar.

En el marco del trabajo de impulsar conductas activas en niños y niñas con sobrepeso y obesidad, focalizados en la intervención "Saltando por su salud", se logró obtener con el grupo experimental y que presentaba sobrepeso y obesidad los mismos resultados positivos en el mejoramiento de la actividad física y la capacidad para superar barreras que se obtuvo con el resto de estudiantes intervenidos. Esto es de suma importancia, ya que que los niños con sobrepeso y obesidad enfrentar mayores problemas en las destrezas motoras y en la percepción de barreras hacia estilos de vida activos, lo que hace de este modelo una opción viable en respuesta a los problemas tradicionales que enfrentan las intervenciones en niños y niñas con sobrepeso, de la dificultad de sostenerlos en el tiempo, al impacto reducido de estos y a los niveles de efectividad cuestionables que se han reportado (Kumanyika, 2001; Goran et al., 1999).

El éxito de esta intervención podría explicarse por varios factores; el uso del mediador de autoeficacia, el mayor tiempo dedicado a la actividad física en las clases de educación física, el desarrollo de destrezas físicas de por vida, la adopción de estrategias conductuales y el desarrollo de entornos propicios para la actividad física. Las estrategias conductuales brindaron, a quienes participaron del programa de intervención, las herramientas para fijarse metas, monitorearse la actividad física y premiarse por los logros obtenidos. Adicionalmente, en el nivel interpersonal, el uso de técnicas de apoyo social por parte de docentes y de la eliminación de barreras complementaron las estrategias del segundo nivel del modelo aplicado. Finalmente, en lo organizacional y de políticas de la escuela, se logró disponer de material y áreas para la práctica de actividades físicas-deportivas durante los recreos. Los tres elementos anteriores enmarcan la intervención ecológica realizada de tercera generación, que otorgó la comprensión de los mecanismos 
mediante los cuales las intervenciones logran el éxito en el cambio de conducta hacia mayores niveles de la actividad física en los niños y niñas (Ward et al., 2007).

\section{Clase de educación física}

Quienes participaron en este estudio mostraron mejorías significativas en sus niveles de actividad física, medida por los pasos realizados; sin embargo, los valores obtenidos son insuficientes para obtener los beneficios que otorga la práctica de actividad física, según las recomendaciones reconocidas para la actividad física para la salud, de 60 min por día; no obstante, el grupo experimental sí logró el valor recomendado de $30 \mathrm{~min}$ en el entorno escolar o el equivalente a 6 mil pasos reportado en diferentes estudios (Colley, Jansen \& Tremblay, 2012; Janssen \& LeBlanc, 2010; Strong et al., 2005; Yetter, 2009).

Aunque los resultados de esta investigación no posibilitan mostrar una tendencia hacia la inactividad física a través del tiempo, sí se podría mencionar que los datos previos a la intervención de las niñas participantes reflejan un alto grado de actitud sedentaria, el cual se modificó positivamente en el grupo experimental en la medición postintervención en las niñas. Este es de una resonancia importante, ya que las mujeres, conforme avanzan en el sistema educativo, mayor es el nivel de sedentarismo que presentan, lo cual coloca a esta población con una conducta tempranera de riesgo para el desarrollo de enfermedades cardio-vasculares, obesidad y diabetes (Pastor et, 2012). Adicionalmente, al igual que esta intervención, Dudley et al. (2011) encontraron que los programas escolares de movimiento humano, que ponen poco énfasis en el área deportiva y se focalizan más en juego, salto de la cuerda, danza y ejercicios aeróbicos, producen mayor participación de estudiantes debido a que producen mayor disfrute.

Al igual que en la intervención llamada SPARK (Sallis et al, 1997), la intervención "Saltando por su salud" logró un éxito evidente en dotar a sus participantes con estrategias y destrezas conductuales que les permitirán desarrollar una conducta activa de por vida (Ward et al., 2007) que es uno de los elementos clave cuando se trabaja con acercamientos ecológicos, que hacen que las intervenciones se dirijan múltiples niveles para causar el mayor impacto (Lox et al, 2006). 
A diferencia de otros estudios en donde se estructuraron intervenciones que modificaron el currículo y las horas dedicadas a educación física (Stone, McKenzie, Welk, Booth, 1998), la intervención "Saltando por su salud" no implicó la inclusión de nuevos elementos en el currículo, ni el incremento en las horas dedicadas a las clases de educación física, solo se ejecutó un reordenamiento de los contenidos en la clase de educación física, estrategias conductuales y el tiempo dedicado a la actividad física, con cambios en los recreos escolares y las políticas institucionales, que permitieron la eliminación de barreras para la práctica de la actividad física. Articular los elementos en una intervención ecológica en salud permitió un refuerzo constante entre cada uno de los contenidos cubiertos; pero, sobre todo, implicó el realizar una intervención con los recursos humanos disponibles y modificaciones en el uso de la infraestructura existente y el del papel docente, brindando grandes resultados, con una gran posibilidad de garantizar la sostenibilidad del desarrollo de escuelas en movimiento.

En este estudio, el diseño de las clases de la educación física para promocionar la participación activa de estudiantes fue una prioridad. Para lograr esto, las clases de educación física lograron dedicar el 50\% del tiempo a actividades de moderada intensidad, junto con tiempo suficiente para el desarrollo de habilidades físicas para la vida, siguiendo un modelo exitoso desarrollado por McKenzie et al. (2010). En términos del tiempo en que el alumnado debe participar en actividad física moderada durante las clases de educación física, no existen directrices en Costa Rica; sin embargo, este estudio tomó como criterio lo expresado por el Centro de Control de Enfermedades y Prevención (2010) y The UK's Association for Physical Education (2008) de recomendar que el $50 \%$ del tiempo de la clase de educación física debe involucrar a sus estudiantes en actividad física moderada a vigorosa.

Los hallazgos de esta intervención se ven avalados por estudios como CATCH (McKenzie et al., 1996) y SPARK (Sallis et al., 1997) que fueron guiados por la teoría del aprendizaje social (Bandura, 1977) y M-SPAN, que se basa en la teoría ecológica (Bronfenbrenner, 1979). La evidencia sugiere que las intervenciones desarrolladas en referencia a una teoría del cambio de comportamiento son más exitosas en el cambio de comportamiento que las que carecen de ese referente teórico (Lubans, Foster \& Biddle, 2008; Michie \& Abraham, 2004). En Saltando por su salud, se propició aumentar el tiempo de aprendizaje activo en 
la clase de educación física, junto con climas motivacionales que destaquen el esfuerzo y la superación personal y proporcionen al estudiantado oportunidades para demostrar el liderazgo y la toma de decisiones (Taylor \& Lonsdale, 2010). Sin embargo, hasta donde se sabe, no hay estudios que hayan investigado el impacto de una intervención basada en destrezas conductuales y metas de logro durante las clases. Las intervenciones que mejoran la motivación hacia las clases de educación física y el incremento en los niveles de actividad física moderada en los estudiantes también pueden aumentar la actividad en el tiempo libre de los niños y las niñas (Chatzisarantis \& Hagger, 2008).

\section{Autoeficacia}

Los resultados positivos obtenidos en la variable de autoeficacia en esta investigación, usando el modelo teórico ecológico, ha dado la posibilidad de predecir el comportamiento activo de sus participantes a través de una disminución de las barreras de infraestructura y humanas. El uso de la teoría de la autoeficacia (Bandura, 1997), incluida en el modelo ecológico, mostró el camino para producir cambios en el factor de barreras para la realización de actividad física en la población infantil de este estudio, lo que lleva a establecer una relación con los cambios en la actividad física voluntaria de sus participantes. Los resultados replican el éxito de la teoría de la autoeficacia encontrados previamente (Annesi, 2006;. Annesi et al., 2007).

Diferentes estudios se han concentrado en el factor de las barreras percibidas que utiliza el constructo de autoeficacia, como con la pérdida de peso (McAuley, Blissmer, Katula, \& Duncan; McAuley, Blissmer, Katula, Duncan, \& Mihalko , 2000), el ejercicio en personas adultas mayores (Dallow \& Anderson, 2003), y el ejercicio en adolescentes (Dischman et al, 2004). Estos estudios establecieron que el incremento de la autoeficacia influyó en los objetivos o metas que el estudiantado estableció, y en la capacidad que desarrolló para persistir en la actividad física a pesar de obstáculos, logrando un incremento en la capacidad para hacer frente a los contratiempos y al estrés y, como tal, directamente influyendo en el compromiso de comportamientos activos. Por ejemplo, Dishman et al. (2004) evaluaron los efectos de una intervención en el entorno escolar, usando estrategias para aumentar la autoeficacia, cambios en la instrucción y modificación en los entornos 
escolares. Los autores encontraron que la manipulación de la autoeficacia tuvo un aumento directo en la actividad física entre participantes.

La ausencia de efectos positivos en los elementos de expectativas y alternativas del constructo de auto-eficacia en esta investigación podría ser explicada por la complejidad de estos factores, en donde la naturaleza, a menudo subjetiva del comportamiento, dificulta las mediciones, particularmente, en la niñez, donde se presentan desafíos específicos para la medición de este constructo y sus compontes en relación con la actividad física debido a sus niveles más bajos de desarrollo abstracto y de funcionamiento cognitivo (Bailey et al., 2005; Pangrazi, 2007).

En la intervención Saltando por su salud, la utilidad predictiva de la autoeficacia, se manejó conservadoramente, sobre todo considerando lo expuesto por Dishman et al. (2004) quien señaló que el aumento de la autoeficacia tiene un efecto directo en el incremento de la conducta de actividad física; sin embargo, la autoeficacia es particular al tipo de actividad que se realiza. Podría ser que el nivel de eficacia para los grupos participantes fuera elevada al realizar ejercicios aeróbicos, pero relativamente pequeña al realizar el entrenamiento con pesas; esta situación podría explicar la falta de resultados positivos en dos de los componentes de la autoeficacia evaluados.

\section{Ambiente escolar}

Al contrario de los modelos tradicionales en promoción de la salud física, que se basan en ambientes totalmente planificados y controlados, con resultados predecibles, que no reflejan el mundo real del comportamiento de la salud física escolar; la intervención Saltando por su salud organizó el entorno del recreo escolar, de tal modo que fueran propicios para la actividad física, entendiendo que el cambio de comportamiento de la salud es un fenómeno complejo y multifacético que tiene múltiples niveles de influencias (Sallis \& Glanz, 2006).

En consonancia con el estudio de Sallis et al. (2001), en este estudio se encontró que la estrategia de ofrecer equipo y demarcar áreas para la práctica de actividad física resultó en un retundo éxito, con efectos más notables en la presencia de supervisión de una persona adulta. Del mismo modo, Fein et al. (2004) encontraron que la disponibilidad de artículos para la práctica de la actividad física y el acceso a las instalaciones deportivas en la escuela se asociaron con mayor reporte de actividad física. 
Los resultados significativos en las variables dependientes, utilizando el mediador ambiental, enmarcan al ámbito de la escuela como el lugar más prometedor para promocionar la actividad física entre los niños y niñas. Este entorno físico es, sin dudas, donde pasan una gran número de horas con oportunidades inmensas para jugar, siempre y cuando se realicen cambios que estimulen a sus estudiantes a hacer actividad física (Kriemler et al., 2011; Timperio, Salmon \& Ball, 2004), como los realizados en este estudio.

\section{Mediador de ambiente}

En el marco de esta investigación, se reconoce que gran cantidad de factores ambientales y sociales que influencian la actividad física en los niños y niñas está fuera de su control, lo que hace que el desarrollo del entorno social, en este estudio, tenga un peso considerable dentro de los resultados positivos obtenidos. Las estrategias o factores de persuasión y de modelaje, usadas por las maestras y el profesor de educación física con el fin de incrementar el apoyo social, responden a investigaciones anteriores sobre la actividad física, las cuales identificaron que el aumento en los niveles de actividad física se asocia con el apoyo social de maestros, familiares y amigos y que los niños y niñas que se dedican a realizar actividad física vigorosa es más probable que animen a otros compañeros y compañeras a ser físicamente activos o activas (Duncan, Duncan y Strycker, 2005; Sallis, Prochaska, \& Taylor, 2000).

Saltando por su salud contó con la participación de la maestra, la cual utilizó el apoyo instrumental y el emocional con sus estudiantes en los recreos. Autores como Willenberg et al. (2010) y Sallis et al. (2001) establecen que la participación de las maestras en el recreo propicia ambientes de paz y de mayor actividad física, especialmente en las niñas. Esto es atribuido a la acción negociadora de las maestras y a la menor dominación por parte de los hombres.

\section{Conclusión}

Esta intervención ha mostrado que el programa Saltando por su salud, con el uso de un modelo ecológico, tuvo un efecto positivo sobre la cantidad de actividad física y la autoeficacia en sus participantes. Adicionalmente, en el marco de la prevención en salud en niños y niñas 
con sobrepeso y obesidad, ha mostrado ser una forma propicia para poder lograr cambios significativos en los patrones de movimiento de los niños y niñas conestas condiciones. Este estudio hace un reconocimiento a la efectividad del modelo ecológico, a la hora de promocionar la actividad física en el entorno escolar, sobre todo porque cambia el paradigma tradicional de ver la realidad desde un punto de vista y no integralmente. Esta vivencia ofrece gran potencial a la hora de desarrollar intervenciones para disminuir los niveles de sedentarismo en niños y niñas, sobre todo cuando se utilizan propuestas basadas en las teorías de las ciencias sicosociales de cambio de conducta y ancladas en la realidad de la escuela pública costarricense.

El programa ha mostrado que los entornos de las clases de educación física y los recreos son los espacios básicos para el incremento de los niveles de actividad física en el contexto escolar, en el tanto se considere como elemento complementario el apoyo social y la organización de la infraestructura en la creación de espacios saludables.

Esta intervención marca el inicio de intervenciones de tercera generación, que representan los primeros esfuerzos de profesionales en educación física por desarrollar intervenciones que contribuyan a promocionar estilos de vida en movimiento en el entorno escolar. Los hallazgos en esta intervención pueden ayudar a entender la conducta sedentaria, pero sobre todo los mediadores que pueden contribuir a paliar esta situación, lo que conduce a darle más relevancia a las teorías y modelos en el cambio de conducta en los niños y niñas, utilizadas en el mundo real. Dichosamente, este estudio da un salto y prueba exitosamente la posibilidad de poder crear entornos educativos en movimiento, utilizando el modelo ecológico.

Se espera que esta intervención sirva de insumo, para que más docentes de educación física y maestros en general se atrevan a implementar propuestas que reviertan la cultura sedentaria de los niños y niñas en el entorno escolar, contribuyendo a paliar la epidemia de sobrepeso y obesidad que se enfrenta. Nuestros niños y niñas desean ser activos, no solo porque es una necesidad básica para su desarrollo integral, sino también porque el jugar es todo para quienes transitan esta edad. 


\section{Referencias}

Aedo, A. y Ávila, H. (2009) Nuevo cuestionario para evaluar la autoeficacia hacia la actividad física en niños. Revista Panamericana de Salud Publica, 26(4), 324-9. Recuperado de http://dx.doi. org/10.1590/S1020-49892009001000006

Annesi, J. J. (2006). Relations of physical self-concept and self-efficacy with frequency of self-selected physical activity in preadolescents: Implications for after-school care programming. Journal of Psychosomatic Research, 61, 515-520.

Annesi, J. J., Faigenbaum, A. D., Westcott, W. L., Smith, A. E., Unruh, J. L. \& Hamilton, F. G. (2007). Effects of the Youth Fit For Life protocol on physiological, mood, self-appraisal, and voluntary physical activity changes in African American preadolescents: Contrasting after-school care and physical education formats. International Journal of Clinical and Health Psychology, 7, 641-659.

Bailey, R. C., Olson, J., Pepper, S. L., Porszasz, J, Barstow, T. J., Cooper, D. M. (1995). The level and tempo of children's physical activities: an observational study. Med Sci Sports Exerc, 27(7):1033-1041.

Bandura, A. (1997). Self-efficacy: The exercise of control. New York: Freeman.

Biddle S. J. H., Gorely, T., Stensel, D. J. (2004). Health-enhancing physical activity and sedentary behaviour in children and adolescents. J Sports Sci. 2004;22:679-701.

Boreham C, Riddoch C. (2001). The physical activity, fitness and health of children. J Sports Sci, 19(12):915-929.

Bronfenbrenner, U. (1979). The Ecology of Human Development: Experiments by Nature and Design. Harvard University Press, Cambridge, MA.

Centro de Control y Prevención de Enfermedades [CDC]. (2015). Calculadora del índice de masa corporal para la población infantil y juvenil. Recuperado de: http://nccd.cdc.gov/dnpabmi/Calculator.aspx

Chatzisarantis, N. L. D., Hagger, M. S., (2008). Effects of an intervention based on self-determination theory on self-reported leisure-time physical activity participation. Psychol. Health, 24, $29-48$. 
Colley, R. C., Janssen, I., \& Tremblay, M. (2012). Daily step target to measure adherence to physical activity guidelines in children. Medicine and science in sports and exercise, 44(5), 977.

Commonwealth Scientific Industrial Research Organisation. (2008). Australian National Children's Nutrition and Physical Activity Survey. In Australian Government Department of Health and Ageing, Department of Agriculture Fisheries and Forestry. Canberra: Australian Food and Grocery Council:1-44.

Cox, M., Schofield, G., Kolt, G. S. (2010). Responsibility for children's physical activity: parental, child, and teacher perspectives. J. Sci. Med. Sport 13, 46-52.

Dallow, B. \& Anderson, J. (2003). Using self-efficacy and a transtheoretical model to develop a physical activity intervention for obese women. American Journal of Health Promotion, 17 (6), pp. 373-381.

Dishman, R. K., Motl, R. W, Saunders, R., Felton, G., Ward, D.S., Dowda, M., \& Pate, R. R. (2004). Self-efficacy Partially mediates the effect of a school-based physical-activity intervention among adolescent girls. Preventive Medicine, 38, 628-636.

Dudley, D., Okely, A., Pearson, P., Cotton, W. (2011). A systematic review of the effectiveness of physical education and school sport interventions targeting physical activity, movement skills and enjoyment of physical activity. Eur. Phys. Educ. Rev, 17, 353-378.

Duncan, S. C., Duncan, T. E., \& Strycker, L. A. (2005). Sources and types of social support in youth physical activity. Health Psychology, 24(1), 3e10.

Fein A. J, Plotnikoff, R. C., Wild C., Spence J. C. (2004). Perceived environment and physical activity in youth. International Journal of Behavioral Medicine, 11(3),135-142. 21.

Giannakidou, D. M., Kambas, A., Ageloussis, N., Fatouros, I., Christoforidis, C., Venetsanou, F., Douroudos, I., \& Taxildaris, K. (2012). The validity of two Omron pedometers during threadmill walking in speed dependent. European of Applied Physiology, 112(1), 49.57. Doi: 10.1007/s00421-011-1951-y

Goran, M., Reynolds, K., \& Lindquist, C. (1999). Role of physical activity in the prevention of obesity in children. International Journal of Obesity, 18-33. 
Holbrook, E. A., Barreira, T. V., \& Kang, M. (2009). Validity and reliability of Omron pedometers prescribed and self-paced walking. American College of Sports Medicine, 41(3) 669-673.

Janssen, I., and LeBlanc, A. G. (2010). Systematic review of the health benefits of physical activity and fitness in school-aged children and youth. International Journal of Behavioral Nutrition and Physical Activity, 7. doi:10.1186/1479-5868-7-40.

Janz K. F., Burns T. L., Levy, S. M. (2005). Tracking of Activity and Sedentary Behaviors in Childhood: The Iowa Bone Development Study. Am J Prev Med, 29(3), 171-178.

Kahn, E. B., Ramsey, L. T., Brownson, R. C., Heath, G. W., Howze, E. H., Powell, K. E., Stone, E. J., Rajab, M. W., Corso, P. (2002). The effectiveness of interventions to increase physical activity: a systematic review. Am J Prev Med, 22, 73-107.

Kriemler, S., Meyer, U., Martin, E., van Sluijs, E., Andersen, L., Martin, B. (2011). Effect of school-based interventions on physical activity and fitness in children and adolescents: a review of reviews and systematic update. Br. J. Sports Me, 45, 923-930. 8.

Kumanyika, S. K. (2001). Mini simposio sobre la obesidad: visión general y algunas consideraciones estratégicas. Annu Rev Salud Pública, 22, 293-308.

Lee, M., Lee, D., Park, C., Park, G., Lee, D., Kim, S., \& Kim, Y. (2012). Validity evidence of Omron pedometer HJ-720ITC for Korean children: A preliminary study. Journal of Science and Medicine in Sport, 15(supp 1), S297.

Lox, C., Martín, K. \& Petruzzello, S. (2006). The Psychology of Exercise. Arizona: HHP.

Lubans, D. R., Foster, C., Biddle, S. J. H. (2008). A reviewofmediators of behavior in interventions to promote physical activity among children and adolescents. Prev. Med, 47, 463-470. 20.

McAuley, E., Blissmer, B. J., Katula, J. \& Duncan, E. (2000). Exercise environment, self- efficacy, and affective responses to acute exercise in older adults. Psychology and Health, 15 (3), 341-355.

McAuley, E., Blissmer, B., Katula, J., Duncan, E. \& Mihalko, L. (2000). Physical activity, self-esteem, and self-efficacyrelationships in older adults: a randomized controlled trial. Annals of Behavioral Medicine, 22, 131-139. 
McKenzie T. L., Crespo, N. C., Baquero, B., \& Elder, J. P. (2010). Leisure-time physical activity in elementary schools: analysis of contextual conditions. J Sch Health, 80, 470-477.

McKenzie, T. L., Nader, P. R., Strikmiller, P. K., Yang, M. (1996). School physical education: Effectof the child and adolescent trial for cardiovascular health. Prev.Med, 25, 423-431.

Michie, S., Abraham, C. (2004). Interventions to change health behaviours: evidencebased or evidence-inspired? Psychol. Health, 19, 29-49.

Organización Mundial de la Salud. (2012). Obesidad y sobrepeso. Nota descriptiva N.311 Recuperado de: http://www.who.int/ mediacentre/factsheets/fs311/es/

Pangrazi, R. (2007). Physical Activity Interventions in Children and Adolescents. Pediatr Exerc Sci, 19(4):493-494.

Peters, B. P., Heelan, K. A., \& Abbey, B. M. (2013). Validation of Omron TM Pedometers using MTI accelerometers for use with children. International Journal of Exercise Science, 2, 106-113.

Salmon, J., Timperio, A. (2007). Prevalence, trends and environmental influences on child and youth physical activity. Med Sport Sci, 50, 183-199. 15.

Salmon, J., Booth, M. L., Phongsavan, P., Murphy, N., Timperio, A. (2007). Promoting Physical Activity Participation among Children and Adolescents. Epidemiol Rev, 29, 144-159. 23.

Sallis, J. F. \& Glanz, K. (2006). "The role of built environments in physical activity, eating, and obesity in childhood", The Future of Children, 16(1), 89-108.

Sallis, J. F., Conway, T. L., Prochaska, J. J., McKenzie, T. L., Marshall, S. J., Brown, M. (2001). The association of school environments with youth physical activity. Am J Public Health, 91, 618-62018.

Sallis, J., McKenzie, T. L., Alcaraz, J., Kolody, B., Faucette, N., Hovell, M. (1997). The effects of a 2-year physical education program (SPARK) on physical activity and fitness in in elementary school students. Sports, Play and Active Recreation for Kids. Am J Public Health, 87(8), 1328-34.

Sallis, J. F., Prochaska, J. J., \& Taylor, W. C. (2000). A review of correlates of physical activity of children and adolescents. Medicine and Science in Sports and Exercise, 32(5), 963e975. 37. 
Stone, E., McKenzie, T., Welk, G., Booth, M. (1998). Effects of physical activity interventions in youth: Review and synthesis. Prev Med, 15, 298-315.

Strong, W. B., Malina, R. M., Blimkie, C. J. R., Daniels, S. R., Dishman, R. K., Gutin, B., Hergenroeder, A. C.,... \& Trudeau, F. (2005). Evidence based physical activity for school-age youth. $J$ Pediatr, 146(6), 732-737. 5.

Taylor, I. M., Lonsdale, C. (2010). Cultural differences in the relationships among autonomy support, psychological need satisfaction, subjective vitality, and effort in British and Chinese physical education. J. Sport Exerc. Psychol, 32, 655-673.

Timperio, A., Salmon, J., Ball, K. (2004). Evidence-based strategies to promote physical activity among children, adolescents and young adults: review and update. Journal of Science and Medicine in Sport, 7 (Suppl 1), 20-29.

Tudor-Locke, C., Bassett, D. R. Jr. (2004). How many steps/day are enough? Preliminary pedometer indices for public health. Sports Medicine, 34(1), 1-8

Tudor-Locke, C., MaClain, J. J., Hart, T. L., Sisson, S. B., \& Washington, T. L. (2009). Pedometry methods for assessing free-living youth. Research Quarterly for Exercise and Sport, 80 (2), 175-184.

Ward, D., Saunders, R., \& Pate, R. (2007). Physical Activity Interventions in Children and Adolescents. USA: Human Kinetics Publishers.

Wareham, N. J., Van Sluijs, E. M. F., Ekelund, U. (2005). Physical activity and obesity prevention: A review of the current evidence. Proc Nutr Soc, 64:229-247

Willenberg, L. J., Ashbolt, R., Holland, D., Gibbs, L., MacDougall, C., Garrard, J., Green, J.,B., Waters, E. (2010). Increasing school playground physical activity: A mixed methods study combining environmental measures and children's perspectives. J Sci Med Sport, 13, 210-216.

Wing, R. R., Goldstein, M. G., Acton, K. J., Birch, LL., Jakicic, J. M., Sallis, J. F. Jr, Smith-West, D., Jeffery, R. W., Surwit, R. S. (2001). Behavioral science research in diabetes: lifestyle changes related to obesity, eating behavior, and physical activity. Diabetes Care, 24(1), 117-123. 4. 
Efectividad del programa ecológico "Saltando por su salud" en la promoción de la actividad física y la auto-eficacia en niños y niñas escolares de tercer grado

World Health Organization. The World Health Report (2002). Reducing Risks, Promoting Healthy Life. Geneva, Switzerland: WHO.

Yetter, G. (2009). Exercise-based school obesity prevention programs: an overview. Psychology in the Schools, 46, 739e 747. 